\title{
Las mujeres bertsolaris, agentes en su incorporación a la escena pública
}

\section{Bertsolari women, agents in their appears into the public scene}

\author{
Gema Lasarte, María Teresa Vizcarra Morales, \\ Andrea Perales Fernández de Gamboa y Vanesa Fernández Rodríguez \\ (Universidad del País Vasco) \\ gema.lasarte@ehu.eus / mariate.bizkarra@ehu.eus / andrea.perales@ehu.eus \\ ORCID: 0000-0002-8362-6142 / 0000-0002-9369-9740 / \\ 0000-0001-6699-101X / 0000-0001-8890-0617
}

\begin{abstract}
Bertsolarism is associated with the masculine and the public. More precisely with the square, a hegemonic place of Basque culture (González Abrisketa, 2013), where the ball is played, politicians speak and improvise and sing the Bertsolaris. Oral improvisation was for centuries natural practice of women and Basque men, but with the changes that took place in the nineteenth century, which prevailed public places - the square - instead of the interiors, women withdrew until they almost disappeared. Not quite, because they timidly made their way into that world of men. This work theorizes about subaltern voices; on the empowerment of Bertsolaris women, who went from invisible to notorious. Also of agency, indispensable in the empowerment of women (Murguialday, 2013; León, 2013). To find out the emergence of the Bertsolaris until the acquisition of the agency in recent decades, 200 questionnaires were passed to these women and interviews were also conducted with 36 of the people surveyed.
\end{abstract}

KEYWORDS: bertsolaris, agency, empowerment, body, improvisation
RESUMEN: El bertsolarismo se asocia con lo masculino y con lo público. Más exactamente con la plaza, lugar hegemónico de la cultura vasca (González Abrisketa, 2013), donde se juega a la pelota, hablan los políticos e improvisan y cantan los bertsolaris. La improvisación oral fue durante siglos práctica natural de las mujeres y de los hombres vascos, pero con los cambios acaecidos en el siglo XIX, que primaron los lugares públicos - la plaza - en lugar de los interiores, las mujeres se retiraron hasta casi desaparecer. No del todo, porque tímidamente se abrieron camino en ese mundo de hombres. Este trabajo teoriza sobre las voces subalternas; sobre el empoderamiento de las mujeres bertsolaris, que pasaron de invisibles a notorias. También de agency, indispensable en el empoderamiento de las mujeres (Murguialday, 2013; León, 2013). Para averiguar la irrupción de las bertsolaris hasta la adquisición de la agencia en las últimas décadas, se pasaron 200 cuestionarios a dichas mujeres y se realizaron, asimismo, entrevistas con 36 de las personas encuestadas.

Palabras-Clave: mujeres bertsolaris, agencia, empoderamiento, cuerpo, improvisación

La existencia de las mujeres bertsolaris ${ }^{1}$ es consustancial al ecosistema cultural vasco, aunque la irrupción de la vida moderna a fines del siglo XIX propiciara su retirada

\footnotetext{
${ }^{1}$ El bertsolarismo se define como el discurso realizado cantando, rimando y midiendo, pero a diferencia de la improvisación que se conoce hoy día en el mundo, en el bertsolarismo se actúa sin la ayuda de ningún instrumento musical.
} 
de la vida pública hasta hacerlas casi invisibles (Larrañaga 1997; Hernández, 2014; Ugalde, 2020). En las últimas décadas, ese paisaje femenino oculto ha ido emergiendo tímidamente, inscribiendo un camino de empoderamiento (Lasarte et al., 2016). En este trabajo abordamos someramente la conceptualización del bertsolarismo y versamos sobre la visibilidad de estas mujeres en la escena pública. En el apartado empírico, combinando herramientas de carácter cuantitativo y cualitativo, indagamos, mediante cuestionarios, la opinión de más de un centenar de mujeres bertsolaris. Asimismo, profundizamos mediante grupos de discusión, relatos y entrevistas en un corpus de 36 personas. Por último, concluimos con los aspectos que han contribuido a la conquista de la agencia, pudiendo adelantar la importancia de las Escuelas de Bertsolaris ${ }^{2}$, donde la mujer bertsolari ha aprendido y ha empezado a cantarlos en un entorno seguro.

\section{APROXIMACIÓN AL CONCEPTO DE BERTSOLARISMO}

Bertsolari, según la etimología, es quien crea bertsos, de estrofas enteras, «artistas de la palabra que improvisan poemas a la vez que cantan siguiendo la melodía de una canción que, muy a menudo, todo el mundo conoce... poemas versificados, rimados y ritmados al momento, cualquiera que sea el tema a tratar» (Laborde, 2005: 11). Lo hace sin el acompañamiento de ningún instrumento musical, a capella, sin contar las sílabas, acomodándolas «a lo que la melodía le pide» (Garzia et al, 2001: 83). Paya (2013) distingue entre el ejercicio improvisado y el escrito, desarrollado con la misma técnica, pero más elaborado pues no se repentiza ante los demás.

El bertsolarismo ha sido considerado parte de la literatura oral (Lekuona, 1978) e improvisada (Lekuona, 1982). Sin embargo, Garzia (2001, 2012) propone un nuevo marco, pues el objetivo de quienes repentizan «no es crear textos literarios de gran valor, sino suscitar emociones entre los oyentes» (2012: 40). Asimismo, la improvisación oral no se agota en el texto, porque también es canto (tono, voz, modo de cantar) y se complementa con el público. Alberdi (2015) afirma que se ha de tener en cuenta lo que siente y piensa el oyente, incluso su desaprobación. Pone de ejemplo al gran bertsolari Xalbador cuando, en la final del campeonato de $1967^{3}$, supo transformar los pitidos del público en fuertes aplausos ${ }^{4}$. Sucedió que muchos oyentes no comprendieron sus creaciones en dialecto bajo-navarro y mostraron su desacuerdo con la decisión del jurado de nombrarle finalista. Para Alberdi, fue el ejercicio más grandioso del bertsolarismo en la gestión de los reproches del público.

No es una producción surgida de la nada, sino una práctica que ha sabido reinventarse, actualizarse y amoldarse a los nuevos tiempos. Aierdi (2007) lo define como una actividad moderna enraizada en la tradición, mayoritariamente aceptada como cultura popular. Pasó de ser una práctica cultural del mundo tradicional a, incluso, un espectáculo de masas actualmente, con sus premios y campeonatos, actividades lúdicas, presencia en la enseñanza reglada, en medios de comunicación, etc.

\footnotetext{
${ }^{2}$ En las escuelas de bertsolaris en la actualidad aprenden a improvisar cerca de dos mil personas con más de cien profesores/as en su mayoría improvisadoras/es. Así en 117 pueblos de Euskal Herria hay una escuela de bertsolaris. Iniciaron su andadura en la década de los 70.

${ }^{3}$ Desde 1935 hasta 2017 se han celebrado 17 campeonatos nacionales entre improvisadores de Baskonia, del Norte y del Sur de los Pirineos. Las fases eliminatorias transcurren durante todo el año, hasta que en la final concurren los ocho mejores, congregando a un público numeroso.

${ }^{4}$ Respondió a los silbidos cantando «... zuek ez bazarete kontentu/ Errua ez daukat ez nik. / Txistuakjo dituzue baina/ Maite zaituztet oraindik» (...si no estáis contentos, / no es mi culpa, no. / Me habéis silbado / pero todavía os aprecio) (traducción de las autoras).
} 


\subsection{Mujeres bertsolaris}

De las mujeres bertsolaris o improvisadoras se sabía muy poco. Se conocían las primeras menciones del siglo XV y las identidades de algunas que vivieron desde fines del siglo XIX (Hernández, 2006; Larrañaga, 1995, 2000). Sin embargo, estudios recientes descubren las identidades de otras mujeres improvisadoras de bertsos o coplas de los siglos XVII y XVIII (Ugalde et al, 2020).

Marginadas en el mundo rural, en los conventos, hasta hace pocas décadas su número era ciertamente escaso. Las mujeres no encajaban en el bertsolarismo moderno, donde brillaban los valores atribuidos a los hombres (Larrañaga, 1995, 2013). Alberdi (2014: 149) asigna características dicotómicas al posicionamiento del bertsolari y la bertsolari respectivamente: «Seguridad, razonamiento firme, competitividad, fuerza, respuesta rápida o gracejo», frente a «dudas, preocupaciones, cuestionamientos, complejos o miedo a ser protagonistas». Esteban (2014: 31) incorpora una imagen de la bertsolari actual muy interesante y habla de agencia, «con un paso hacia atrás, atenta, en alerta esperando a subir al escenario». Una imagen que subvierte la identidad androcéntrica del discurso imperante.

La pionera del bertsolarismo contemporáneo fue Cristina Mardaras, cuando participó por primera vez en un campeonato (1985). Arantzazu Loidi, compañera de escenario, fue criticada por su voz, demasiado fina para los estándares que exigía la masculinidad hegemónica. Otras mujeres tampoco se amedrentaron: Iratxe Ibarra, Estitxu Arozena, Nerea Elustondo, Amaia Agirre o Maialen Lujanbio, campeona nacional en 2009 y 2017 . Hubo quien no saltó a la escena pública o lo dejó a medio camino. Todas auspiciaron la reflexión que sacudió el bertsolarismo y favorecieron la incorporación de la visión de género en aquel mundo de hombres (Larrañaga, 2013).

\subsection{Empoderamiento, agencia y cuerpo}

Otro aspecto clave del bertsolarismo es la ausencia de artificios cuando sus protagonistas actúan ante el público, siendo lo más habitual tomar asiento y levantarse cuando quien dirige el evento requiere su intervención. En ese ritual las mujeres han ido cambiando su manera de exponerse en el escenario al lado de los hombres. Al respecto, Hernández (2014) comenta que en el bertsolarismo contemporáneo apenas ha habido lugar para la feminidad; sí para las mujeres, pero desde la performatividad de la masculinidad. Habla de travestismo y de imitar a los hombres.

Alberdi (2014, 2015), refiriéndose al binomio bertsolari-cuerpo, señala dos generaciones de bertsolaris modernas. Las Drag King, que emularon cuerpos masculinos en sus posturas, su forma de vestir o de cantar; y las Panpinak o muñecas, que han transgredido los cánones masculinos con sus maquillajes, escotes y minifaldas. La generación de las Drag King fue la de las precursoras de la década de los 90, mujeres atrevidas, decididas y fuertes, con agencia, que se acomodaron al estilo vigente y lograron ser valoradas cual bertsolaris varones. Ahora predominan las Panpinak, más femeninas, que cumplen con los parámetros de la heterosexualidad y del rol femenino inscrito en ese orden.

Estas dos formas de aparecer ante el público de las bertsolaris reflejan el cambio fraguado en el bertsolarismo como marca cultural. El proceso de empoderamiento y visibilidad de las bertsolaris corre paralelo al desarrollo del bertsolarismo más cultivado, urbano; a la incorporación del público femenino; al nuevo perfil de los y las bertsolaris; al cambio de la temática propuesta en las improvisaciones y, sobre todo, a la aparición de

\footnotetext{
${ }^{5}$ Para más información de la historia de las bertsolaris, Ugalde et al. (2020).
} 


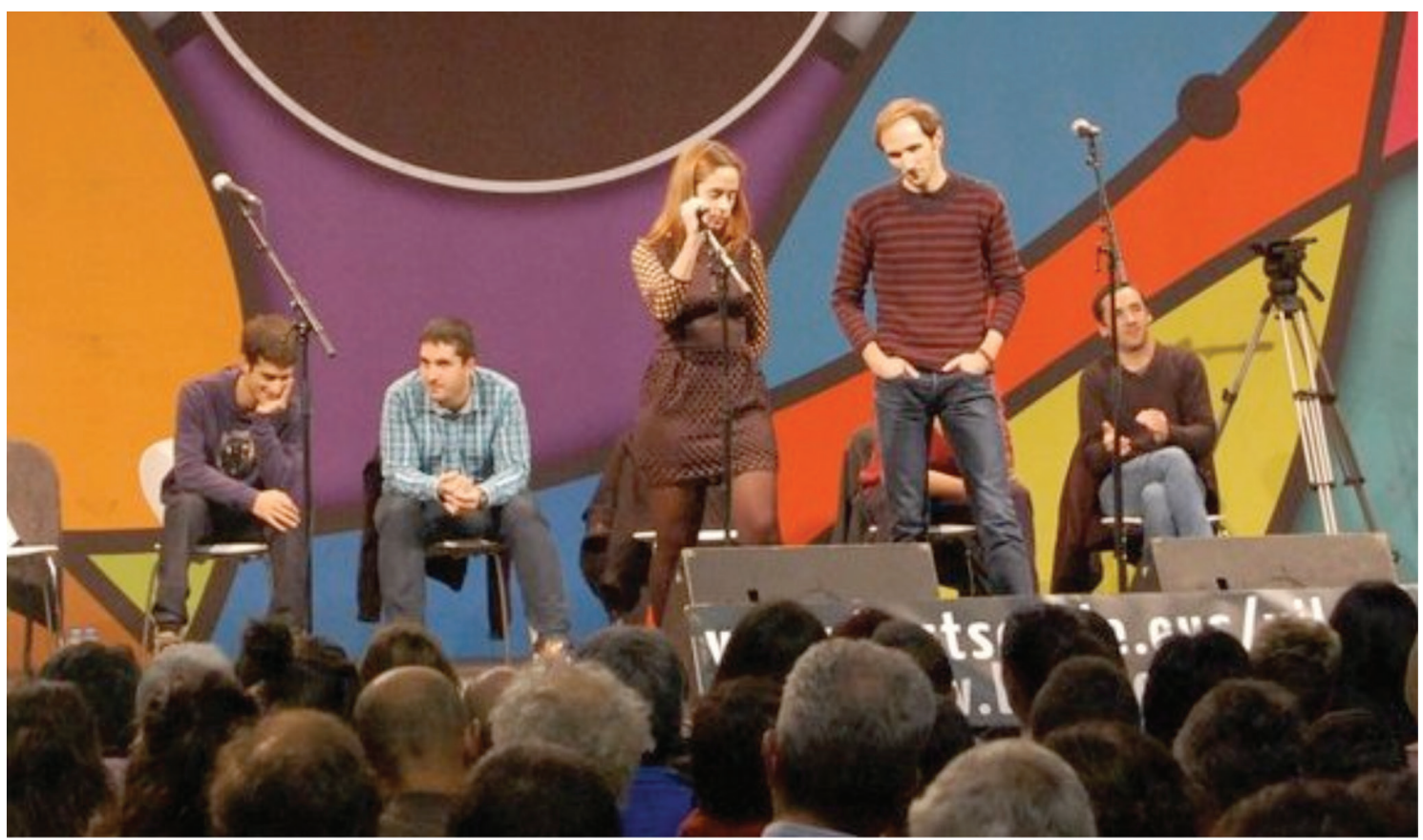

Figura 1: Maddalen Arzallus y Sustrai Colina improvisando en Bayonne (15-11-2014). Sentados, Unai Barroso, Eneritz Zabaleta, Xumai Murua y Ramuntxo Christy. Fuente: Xenpelar Dokumentazio Zentroa.

las Escuelas de Bertsolaris, donde se han formado las nuevas generaciones, sean hombres o mujeres (Lasarte et al, 2016).

Al hablar de empoderamiento, nos referimos a las relaciones de poder existentes (Foucault, 1972; Gramsci, 1971; Freire, 1986), de desafiarlas y obtener un mayor control sobre sus fuentes (Batliwala, 1997 in Murguialday, 2013). También del compromiso de alterar radicalmente los procesos y estructuras que reproducen la posición subordinada de las mujeres (Oakley, 2001). El empoderamiento concierne al colectivo desempoderado que ha de ganar poder (Murguialday, 2013).

Es interesante la interpretación tripartita de poder confeccionada por Lukes en Power. A radical view (1974). Discierne tres caras diferentes del mismo: el visible, el oculto y el invisible. El poder visible se vale de expresiones formales, de las reglas de juego explícitas de la sociedad. El poder oculto se erige en las reglas y procedimientos que vuelven invisibles a las voces subalternas (intimidación, coerción, desinformación). Por último, el poder invisible fomenta las prácticas y las normas culturales que disuaden a la gente de actuar para el cambio. Así, Lukes nos sitúa en diferentes niveles de análisis (global, regional, nacional, familiar...) y en distintas esferas (física, socio-cultural, religiosa, política...) (León, 1999). Gracias a esta especificación, se focaliza mejor el lugar de subordinación de la mujer y, por ello, nuestra investigación la ubicamos dentro de los poderes ocultos e invisibles y en la esfera socio-cultural.

Para contrarrestar las tres formas de poder y afrontar la exclusión, Venklasen y Mille (Murguialday, 2006) plantean distintas estrategias. Contra el poder visible, para cambiar la realidad, enfatizan en la necesidad de ganar un poder que atañe a la agencia. Murguialday (2013: 37) afirma que «para acceder a esta dimensión política del empoderamiento, las mujeres han de actuar colectivamente en la arena pública, tomando parte en todos los ámbitos donde se toman decisiones relevantes para ellas y sus colectivos de pertenencia»». 
Con el fin de desafiar al poder oculto, Mosedale (2005) percibe la necesidad de que las mujeres puedan transitar del «yo» al «nosotras», y habla necesariamente de la identidad colectiva. Ésta se forjará con condiciones favorables, espacios sociales donde las mujeres puedan sentirse seguras y valoradas; redes que puedan permitir expandir las nuevas visiones, y respuestas sociales a su nueva identidad que fuercen a cada mujer a confrontar las creencias heredadas (Evans in Murguialday, 2013).

Para posicionarse frente a la invisibilidad, la mujer necesita ganar poder personal, debe fortalecerse a sí misma y ser consciente de que vive subordinada. La reflexión sobre su sometimiento será el paso inicial hacia el empoderamiento. Primero, será una labor particular; después, este poder interno se socializará y se compartirá mediante el reconocimiento e integración de las experiencias internas y externas. Finalmente, se hablará de la capacidad de incidencia política y del poder para el cambio.

Todo este proceso de empoderamiento concierne a la agencia y a las opciones que se construyan. Kabeer (1999) relaciona la agencia con la capacidad de hacer elecciones vitales estratégicas, en la capacidad de ver que hay alternativas a lo establecido. Es decir, que las voces subalternas vean claramente su situación de subordinación y la posibilidad de cambio (Spivak, 1993). La agencia, por tanto, puede ejercerse de forma individual o colectiva. Puede expresarse como negociación, manipulación, subversión y resistencia. Esta definición coincide con el término de agency que utiliza Ortner (2006). En este trabajo entendemos la agencia como acción social e individual. Esteban (2004) nos propone ver a las mujeres como agentes de sus vidas, en lugar de víctimas. Asimismo, según afirmaba Haraway (1995), nos interesa hablar del cuerpo como agente, que significa al mismo tiempo construir y destruir máquinas, identidades, categorías, relaciones e historias del espacio.

Kabeer (1999) señala tres ámbitos donde se pueden evaluar los cambios de agencia: la participación en la toma de decisiones, la movilidad en el ámbito público y la violencia masculina. En el primero, la agencia de las mujeres mejora notablemente cuando éstas tienen voz para expresarse sobre aspectos críticos de su vida, o sobre temas que fueron vetados en el pasado. No sólo importa el acto de decidir, también importa la trascendencia de las áreas en las que se decide. Otro indicador se da en relación a las localizaciones, de tal forma que la movilidad de las mujeres se convierte en una cartografía de poder o subalternidad, porque existen lugares prohibidos o inadecuados para ellas, un continuum entre los lugares aceptables y no aceptables para las mujeres. Por último, refiere que los datos cuantitativos en relación a la violencia masculina ${ }^{6}$ sugieren que «las mujeres de mayor edad, con hijos varones, con nivel educativo o que aportan ingresos al hogar tienen menor probabilidad de violencia que las jóvenes, sin hijos varones, educación e ingresos» (Murguialday 2006: 27).

En relación a la investigación que abordamos, aparecen algunos ámbitos como claros indicadores de agencia. Primeramente, porque la bertsolari abandona el espacio privado a la conquista de lo público, del escenario. En segundo lugar, porque en el transcurso del tiempo cambia su perfil personal. En este transitar desde el «poder sobre» al «poder para» y al «poder con» nos hemos detenido en sus itinerarios corporales, en el cuerpo como indicador de agencia, y hacemos nuestra la definición de Esteban (2004: 43) «de que el cuerpo es un nudo de estructura y acción, de experiencia y economía política». El cuerpo es entendido como el lugar de la vivencia, el deseo, la reflexión, la contestación y el cambio social. La centralidad del cuerpo se refleja en el mundo

\footnotetext{
${ }^{6}$ Entendida, en este caso, como violencia simbólica (Bourdieu, 2000; Lasarte, 2012).
} 
del espectáculo, del ocio y del deporte. De ahí, la importancia de la identidad corporal en el bertsolarismo, a la que se responde con el aprendizaje de distintas técnicas. Por ello y relacionado con este empoderamiento, este artículo tiene como objetivos:

1. Conocer el perfil de las actuales improvisadoras vascas

2. De las características que reúnen las mujeres bertsolaris encuestadas, detectar los elementos que son o pudieran ser facilitadores de agencia.

3. Conocer qué piensan ellas que en un futuro puedan ser elementos facilitadores de agencia en el camino del empoderamiento

4. Tomando el cuerpo como agente, ver qué itinerarios corporales han elaborado las mujeres entrevistadas en su recorrido como mujeres del espectáculo.

5. Tomando el cuerpo como agente, investigar cómo el cuerpo les ha ayudado a empoderarse en las últimas décadas.

\section{Metodología}

Esta investigación se planteó como un estudio de caso, con el fin de aproximarnos al perfil de las actuales bertsolaris, sus características, sus itinerarios personales, cómo su cuerpo ha influido en sus respectivos empoderamientos. Primeramente, se contactó con 200 mujeres bertsolaris mediante correo electrónico, para enviarles y se les envió un cuestionario diseñado en Google Forms. Dicho cuestionario comprendía, en su primera parte, preguntas cerradas concernientes a la edad, estudios y profesión. En la segunda, se interrogaba sobre la formación, experiencia y vivencias relacionadas con el bertsolarismo y el género. Se incidió en las Escuelas de Bertsolaris, ya que se afirma que sin las mismas no podría hablarse hoy de mujeres bertsolaris (Artetxe, 2016). También se les preguntó sobre su experiencia en el espacio público y sobre los aspectos que creían oportuno ahondar para que en el futuro la presencia de las mujeres en la improvisación oral vasca se fortalezca de forma sistemática y simétrica. Se recibieron 121 cuestionarios y se analizaron los resultados con el programa SPSS.22.

Para profundizar en el aspecto corporal, elemento ausente en la historia del bertsolarismo, se realizaron cinco grupos de discusión (GD), se recogieron cinco relatos escritos (R), y se realizaron 10 entrevistas individuales en profundidad a bertsolaris de élite (EP), siguiendo un guión de trabajo previo, elaborado en la misma línea de los cuestionarios. En esta segunda parte de la investigación participaron 36 bertsolaris, hombres y mujeres, entre las que se hallaban las más conocidas de la escena vasca (Tabla 1). En los códigos de los GD y R se ha incorporado la fecha de la entrevista y, al final, el sexo ( $\mathrm{H}$ o $\mathrm{M})$, en las EP se ha integrado el número de la bertsolari. Berts1 20151112; Berts2 20151119; Berts3 20151201; Berts4 20151201; Berts5 20151203; Berts6 20151224; Berts7 20160104; Berts8 20160109; Berts9 20160115 y Berts 1020160131 . El uso de estas herramientas, como parte del estudio de caso, fue fundamental para registrar «la conducta de las personas involucradas en el fenómeno estudiado» (Martínez, 2006: 167), sus vivencias, sentimientos y pensamientos, recopilando las diferentes acepciones que hay en torno al bertsolarismo y al género desde un planteamiento comunicativo y dialógico (Habermas 1994; Aubert et al, 2009).

Los resultados de los grupos de discusión, relatos y entrevistas se analizaron mediante el programa NVIVO11, tomando como eje el cuerpo femenino sobre el escenario, cómo lo han vivido ellas y qué es lo que otros dicen del mismo. 
[TABla 1: PARTicipantes EN LOS GRUPOS DE DisCusión, RELATOS Y ENTREVISTAS]

\begin{tabular}{|l|l|c|c|c|}
\hline \multicolumn{1}{|c|}{ Grupo } & \multicolumn{1}{|c|}{ Individuales } & Número de participantes & Mujeres & Hombres \\
\hline G1 & & 3 & 3 & 0 \\
\hline G2 & & 5 & 5 & 0 \\
\hline G3 & & 6 & 3 & 3 \\
\hline G4 & & 4 & 4 & 0 \\
\hline G5 & & 3 & 2 & 1 \\
\hline & R (relato) & 5 & 5 & 0 \\
\hline & $\begin{array}{l}\text { EP (entrevista en } \\
\text { profundidad) }\end{array}$ & 10 & 10 & 0 \\
\hline
\end{tabular}

3. REsultados

3.1. El perfil de las mujeres bertsolaris

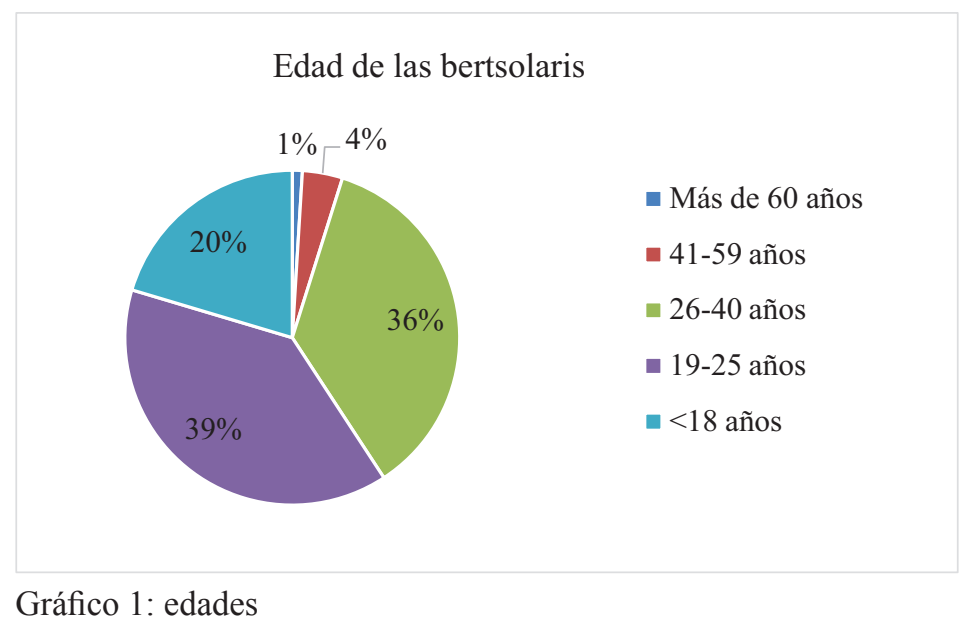

Tal y como vemos en el gráfico 1, y si atendemos a la edad, las bertsolaris son mujeres jóvenes. Salvo un 1\% que supera los 60 años y un 4\% de entre 41 y 59 años, las demás se hallan por debajo de esta franja. Así, el 36\% corresponde a las que tienen entre 26 y 40 años, con un 39\%, entre 19 y 25 años, hasta alcanzar el 39\% para las menores de 18 . Son, pues, muy pocas las que llevan compitiendo algún tiempo, ya que se inician

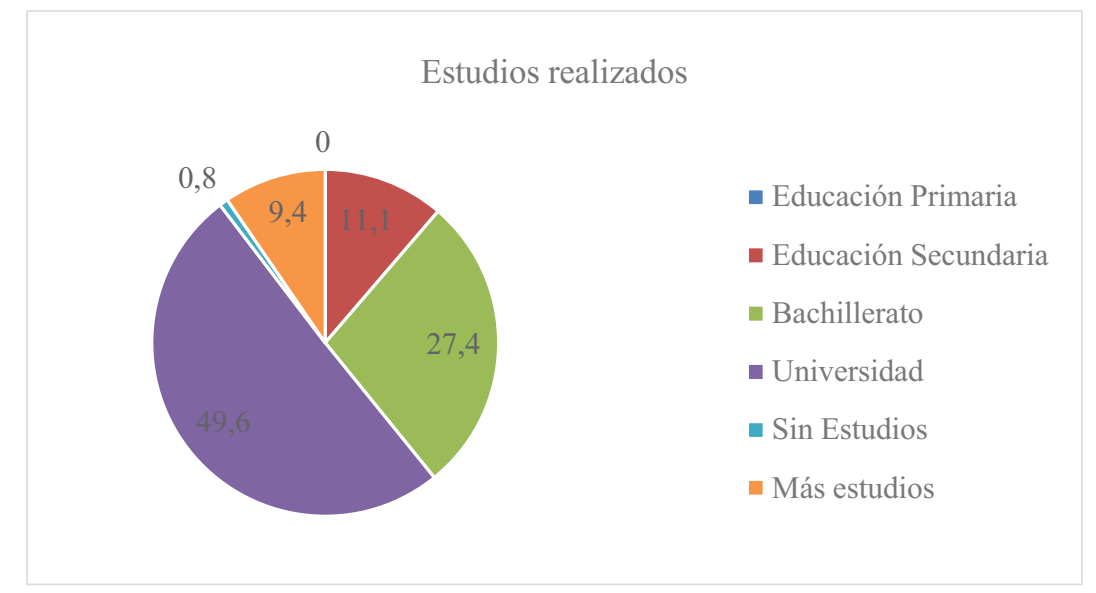

Gráfico 2: estudios 
en ese mundo con 15-20 años. Al ser la mayoría tan joven, es fácil pensar que son más vulnerables a la asimetría de género. Sin embargo, su formación académica nos lleva a deducir que poseen conocimientos y recursos para la vida.

Como lo podemos ver en su correspondiente gráfico (gráfico 2), la juventud se hace notar en su nivel académico, pues casi la mitad es universitaria (49,6\%), amén del 9,4\% que posee más estudios, el 27,4\% cursa el Bachillerato, junto al 11,1\% que se halla en Secundaria.

\section{Profesiones de las bertsolaris}

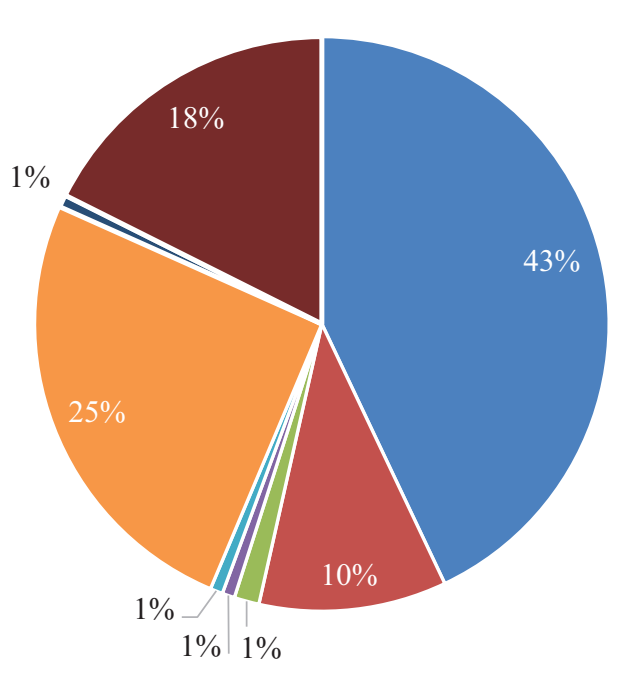

- Otras profesiones

- No responde

- Profesora de bertsolaris

- Trabajadora en Amigos del Bertsolarismo y profesora

- Escritora y bertsolari

- Estudiante

- Estudiante y monitora de tiempo libre

Gráfico 3: profesiones

Entre las profesiones que ejercen las bertsolaris (ver gráfico 3), la mayoría se gana la vida en trabajos sin relación con el bertsolarismo (34,43\%). Destacan las estudiantes $(29,8 \%)$

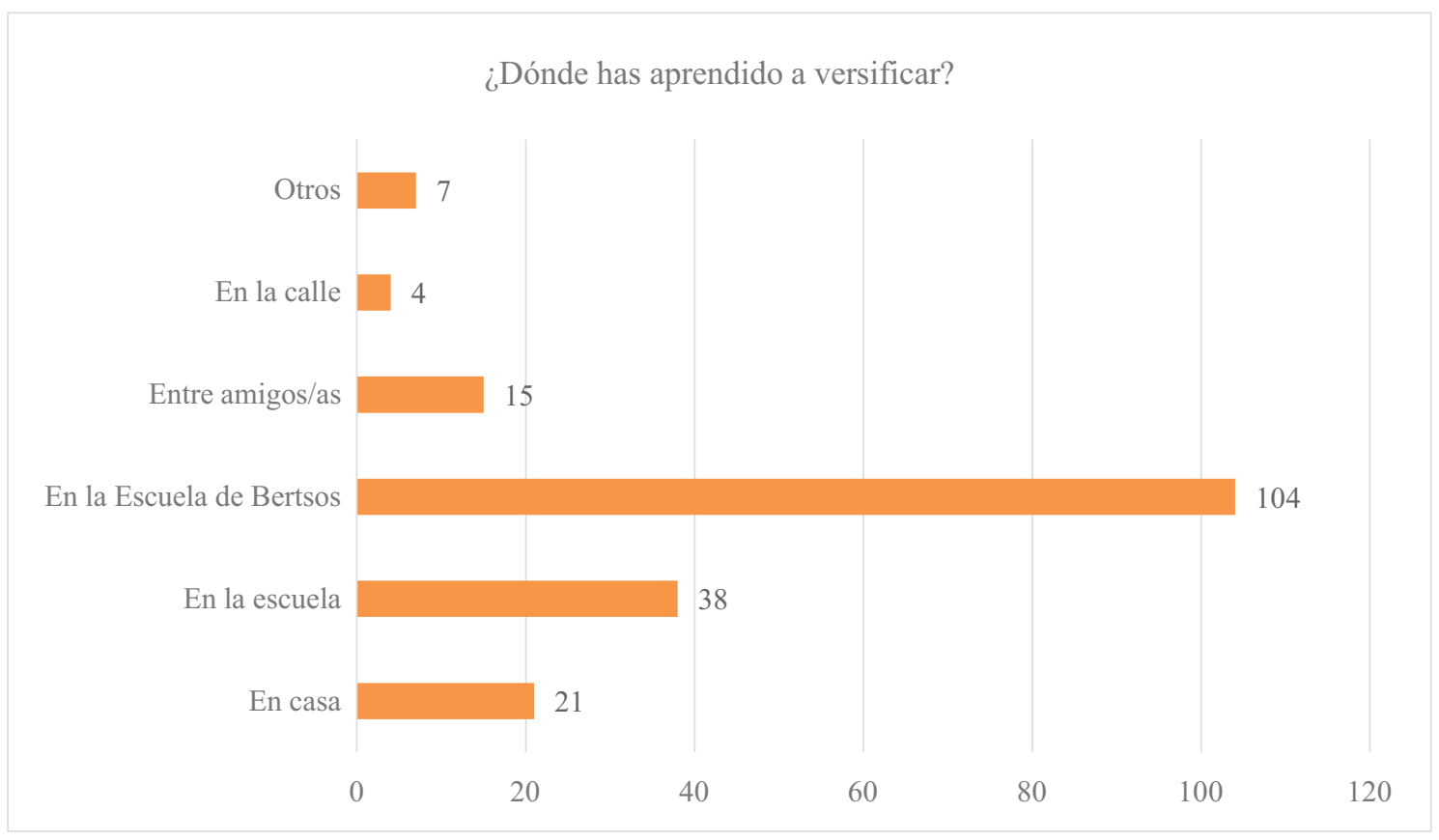

Gráfico 4: encuesta 
y las profesoras (20,7\%). Son residuales la escritora, la que trabaja en una Asociación de Amigos del Bertsolarismo y es profesora de bertsolaris, y la estudiante y monitora de tiempo libre $(0,8 \%$ respectivamente), así como las 2 que son profesoras de bertsolaris $(1,7 \%)$. Un $12,4 \%$ no respondió a la cuestión.

Es interesante despejar la cuestión de dónde aprendieron a versificar. El gráfico 4 demuestra que la mayoría (57\%) lo ha hecho en las Escuelas de Bertsolaris y la quinta parte $(21 \%)$, en la escuela. En el ámbito familiar, el 12\%; junto con las que se han ejercitado entre colegas $(8 \%)$ o en la calle $(2 \%)$. Los espacios seguros han sido, pues, decisivos en el aprendizaje del canto improvisado.

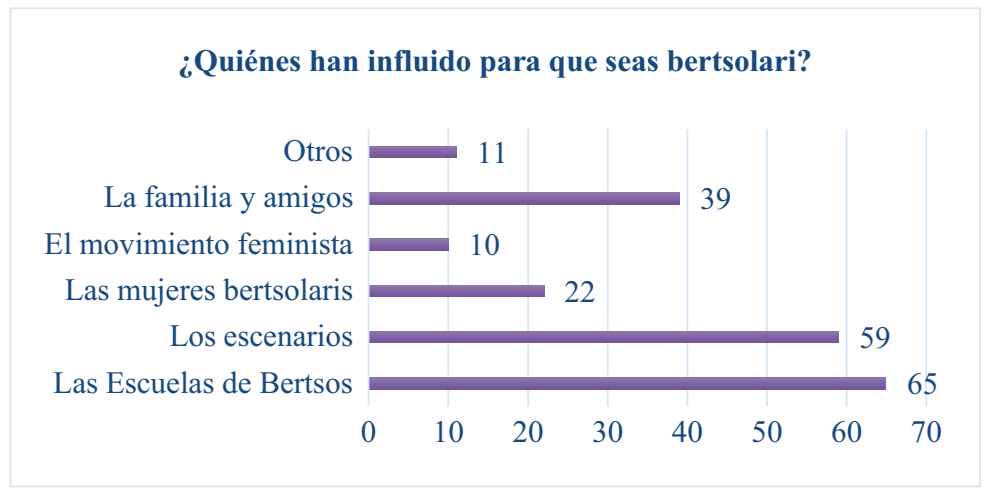

Gráfico 5: influencias

Interrogadas sobre quiénes han influido para que sean bertsolaris (ver gráfico 5), las Escuelas de Bertsolaris se alzan con la mayoría, pues 65 mujeres respondieron afirmativamente. Les sigue el gusto por el escenario (59), la familia y amigos (39), otras mujeres bertsolaris (22), el movimiento feminista (10) y 11 no especificaron ninguna razón.

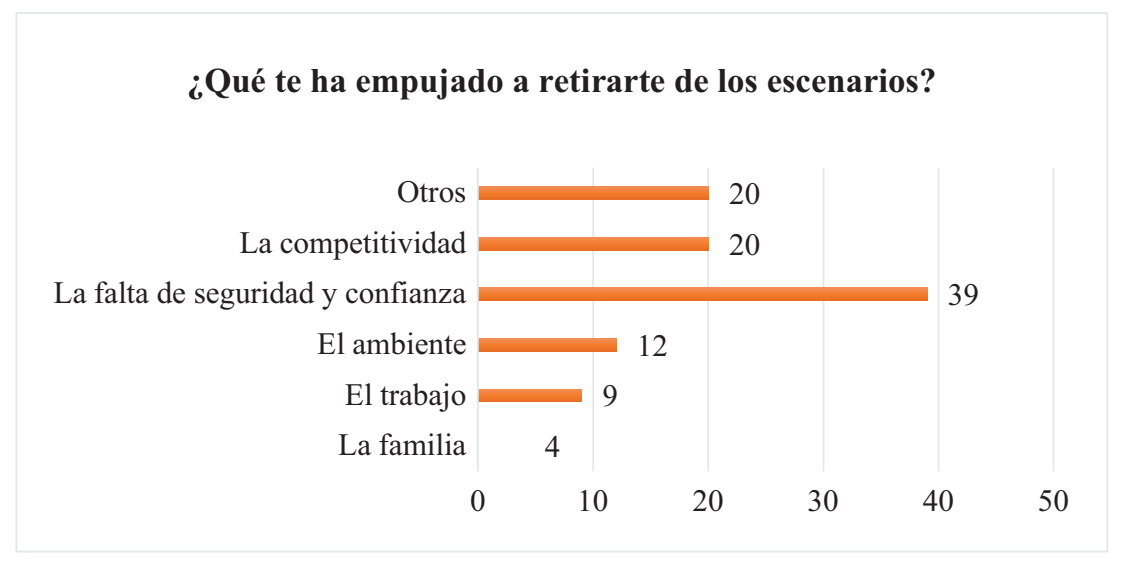

Gráfico 6: retirada de escenarios

También quisimos preguntarles por qué se retiran del espacio público, cuál es la razón de su abandono. Llama la atención, como se puede observar en el gráfico 6, que las mujeres inscritas en las Escuelas de Bertsolaris alcancen el 48\%, mientras que en la esfera pública sólo sean el 20\%. ¿Qué ocurre para que las mujeres, pese a estar preparadas para improvisar, no compartan escenario con los bertsolaris? 
Las razones esgrimidas son diversas. Es mayoritaria la falta de seguridad y confianza (39), posiblemente lo que llamamos miedo escénico por su perfeccionismo exacerbado; la competitividad (20), el medirse constantemente con otros en las tablas, tampoco es baladí; el ambiente (12); razones profesionales (9) y la familia (4). 20 bertsolaris no especifican las causas.

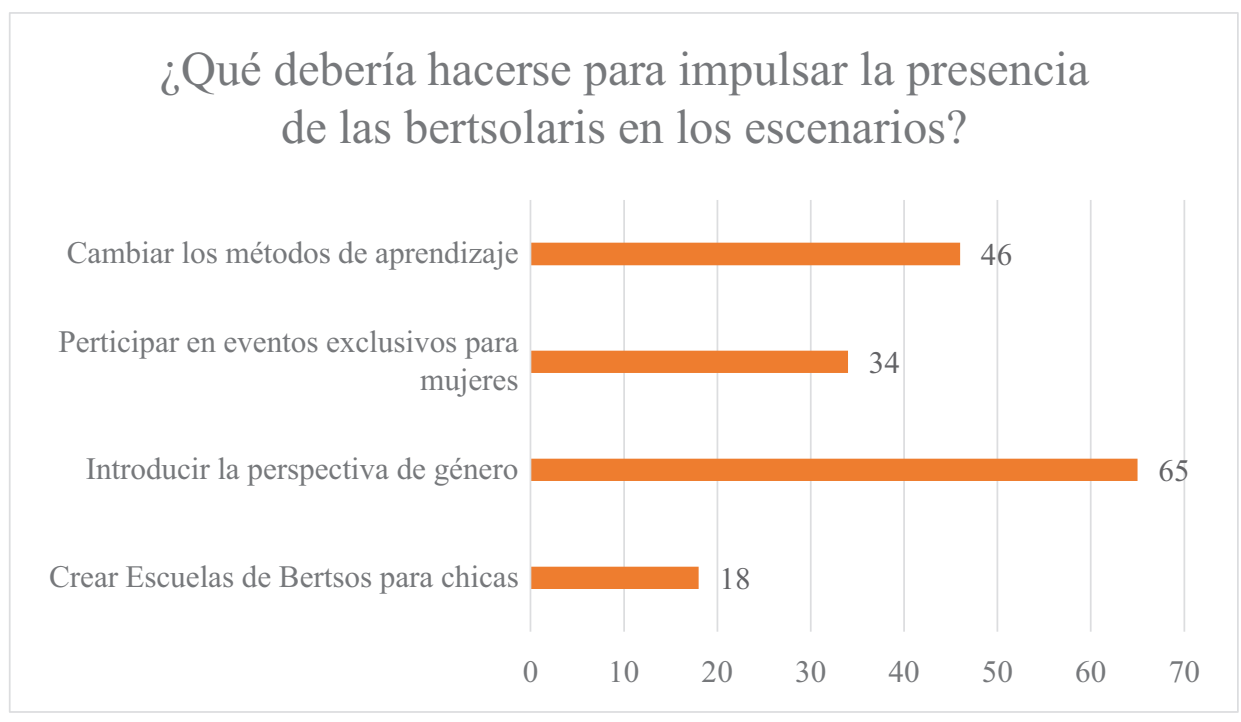

Gráfico 7: iniciativas propuestas

Fueron 103 bertsolaris las que respondieron a la cuestión de lo que habría de hacerse para romper con esa tendencia, para impulsar la presencia de las mujeres en el espacio público, tal y como se demuestra en el gráfico 7. 65 esgrimen la introducción de la perspectiva de género, seguidas por las que propugnan un cambio en los métodos de aprendizaje (46). Medidas más específicas son las que defienden los eventos exclusivos para mujeres (34) o las Escuelas de Bertsolaris femeninas (18).

Los datos fríos y escuetos obtenidos de las encuestas, deben ser matizados y enriquecidos con los obtenidos en los grupos de discusión, relatos y entrevistas, donde el cuerpo se erige en cuestión principal.

\subsection{La presencia del cuerpo en el escenario}

Sometidas al escrutinio del público, acostumbrado a los bertsolaris varones, las pioneras atestiguan que de forma inconsciente lo tapaban, borrando sus rasgos femeninos. Su objetivo era cantar como si no lo tuvieran, como si sólo cantara el intelecto, porque el bertsolarismo se sustenta en la disputa dialéctica (Alberdi, 2015).

Llevabas camisas amplias. Pero esto no sólo ha ocurrido en el bertsolarismo, es decir, que cuando la mujer ha intervenido en espacios masculinos, lo que ha querido es que nadie se fijara en ella y que la competición se centrara del cuello para arriba (150109_GD1_M).

Más aún, algunas recuerdan cómo en las Escuelas de Bertsolaris, donde aprendieron a cantar en público, sus profesores les instaban a comportarse como a los chicos, «firme y canta alto» (20160115_ Berts9_M), «saca pecho», «adelante», «no te apoques» (20151201_Berts3_M), precisamente cuando trataban de disimular sus senos incipientes. 
Incluso Maialen Lujanbio, campeona de 2009 y 2017, se pregunta si fue casualidad que las que perduraron en la arena pública, las Drag King, respondieran a unas características definidas: cuerpo y voz fuerte, contundente, respuesta rápida, descaradas (Alberdi, 2015). Una compañera (20151201_Berts3_M) recuerda que a ella de joven le alababan por su voz, aspecto físico e ímpetu, con afirmaciones como "¡vaya huevos tiene!» y recuerda que a las más agraciadas, sin reparar en sus cualidades bertsolarísticas, les dedicaban expresiones como "iqué chica más guapa». Algo que no sucede con «los chicos apuestos y "femeninos" que siempre van de punta en blanco, presumidos y muy sensibles», a los que nunca se les dice nada parecido. "A nosotras siempre nos lo recalcaban».

No parece que fuera una opción consciente, por lo menos para la mayoría. Las bertsolaris de ayer, hoy y mañana son mujeres de su tiempo. Así nos lo cuenta una precursora:

No creo que nosotras reflexionáramos en torno a nuestro cuerpo, en torno a nuestro estilo, sobre nuestra actitud. Ni nosotras ni las nuevas generaciones. Con 18-20 años, ¡dichosas nosotras si lo hubiéramos hecho! Nadie lo ha hecho. Nos ha pillado nuestra época, la izquierda radical vasca, la sociedad, el mercado. Y funcionamos según esos mecanismos. (150109_GD1_M).

No parece que las nuevas generaciones hayan sentido la necesidad de ocultar su feminidad. Al contrario, la reivindican maquilladas, con sus vestidos ajustados y tacones. Alberdi (2015) afirma que las llamadas muñecas o Panpinak lo han tenido más fácil, pero que se les exige que sean moderadas, para ser aceptadas por el patriarcado. Hay quien no se reconoce como tal, siendo como fue «de las primeras en cantar con minifalda y tacones con 15 años». Considera el cambio estético como generacional y que no afecta exclusivamente a las bertsolaris, que el canon estético-político de cada época marca una tendencia y que vestir con aires masculinos fue una moda que identificó a cierto sector de la juventud vasca (20160131_Berts10_M).

La forma de aparecer y actuar en público, dicen que la marca el mercado. Obviamente a un organizador le importa el auditorio, cuando elige quiénes van a concurrir en cada recital, sean hombres o mujeres. Al lado de las grandes estrellas, parece que el gran público aprecia a las bertsolaris que cuidan su aspecto.

No es ninguna casualidad que las bertsolaris que al año hacen 50 o más de 70 recitales, casi todas, por no decir todas, sean muy arregladas (1501031_GD2_M).

Atinadamente una informante (20160104_Berts7_M) señala que no existe un solo público, que cada recital es único y diferente y que las mismas bertsolaris se adecuan al mismo, que «no se visten de la misma manera si cantan en Sanfermines o en el Gaztetxe ${ }^{7} »$.

Aun así, las bertsolaris observan que su estética es a menudo utilizada por sus compañeros en intervenciones que, en principio, no guardan ninguna relación con la misma, visibilizándose la subordinación de los cuerpos femeninos.

Ahora las bertsolaris aparecen de forma natural, con formas y estilos que responden a lo que se ve a diario, pero no sin costes. $Y$ es que esta demostración de feminidad las convierte en constante tema a tratar en boca de los bertsolaris (150109_GD1_M).

\footnotetext{
${ }^{7}$ Lugar donde se reúne la juventud para realizar diversas actividades.
} 
Son conscientes de que, cuando se hallan en el escenario, el cuerpo también canta, desde los dedos de los pies hasta los pelos de la cabeza. Cada una es su cuerpo, y cada una tiene que vivir lo mejor que pueda con el mismo, sin dejarse presionar por los gustos externos.

Los cuerpos hablan y no los tenemos que silenciar. Cada una se tiene que reconciliar con su cuerpo y con su modo de expresión corporal, tiene que saber quién es y quien parece que es. Y aceptarlo (150305_R5_M).

Saben que la complexión fuerte y grande puede emanar seguridad y un cuerpo delgado y pequeño, fragilidad. Lo mismo sucede con la voz demasiado fina de la mayoría de las mujeres. Por eso, aunque sean atributos que pueden propiciar la desaprobación externa, también pueden transformarse en armas de empoderamiento. Cuanto más se conozcan y se acepten, tendrán más recursos para improvisar en público. Dicen que, al estrado, a la plaza van tal cual son, con su cuerpo. Pero muchas veces sucede que lo mental se sobrepone a lo físico, difuminando su cuerpo, porque eso es lo que han aprendido en las Escuelas de Bertsolaris, a cantar con el intelecto, obviando sus cuerpos.

¿Dónde está el empoderamiento del cuerpo? Viendo la fragilidad de esos cuerpos grandes y fuertes y la fortaleza de esos cuerpos pequeños y delgados. Tenemos dónde aprender. Todavía yo no he aprendido que, además de mi mente, tengo que incorporar el cuerpo en el bertso, porque me sumerjo solamente en la creación desde la base del intelecto, porque desde pequeña he aprendido a cantar y a relacionarme con el bertso en dialéctica con el intelecto, y no con el cuerpo (150305_R5_M).

A pesar de toda la problemática que las acompaña, consideran estar viviendo un momento muy interesante. Les resulta hermoso ver en sus «compañeras diferentes maneras de ser mujer, de ser bertsolari, de manifestarlo en la plaza» (20151112_Berts1_M).

\section{Conclusiones}

Las mujeres improvisadoras vascas actuales son mayoritariamente jóvenes, como también es reciente el fenómeno de la aparición o, mejor, la visualización de la mujer en el bertsolarismo. El 40\% de las mujeres está en la franja de 19 a 25 años, un 37\% en la de 26 a 40 y solo el 4\% en la de 40 a 60 años. Además de este aspecto, hay que destacar su nivel de formación, puesto que el $49,6 \%$ posee estudios universitarios y otro 27,4 $\%$ ha cursado el Bachiller, comprensible, pues el $20 \%$ es menor de 18 años. Otro dato importante es el concerniente a su independencia económica, ya que el 70,8\% trabaja y otro $20,7 \%$ estudia. Como primera conclusión, podemos afirmar que las bertsolaris vascas de hoy en día son mayoritariamente mujeres jóvenes, con estudios universitarios e independientes. Su perfil muestra claros indicadores que favorecen la agencia. Por una parte, por su alta cualificación formativa y por otra, por su poder adquisitivo, gracias a que poseen un trabajo remunerado, aspectos importantes que derivan en la adquisición de la agencia (Kabeer 1999).

Siguiendo a Kabeer y en relación a su empoderamiento, aparte de reforzar su estado interno, su situación personal, la mujer necesita trasladar esa identidad al colectivo, a la sociedad, y para ese tránsito necesita redes y zonas asequibles y seguras (Murguialday 2013). Las mujeres bertsolaris lo han hallado en las Escuelas de Bertsolaris, donde se ha iniciado en el bertsolarismo el $88,9 \%$ de las mismas. La mayoría ha adquirido técnicas, 
ha desarrollado su creatividad y ha aprendido a cantar en público en estas escuelas. El $48 \%$ del alumnado de estas escuelas son mujeres (Erkiaga, 2008) que eligen formarse libremente. Son lugares seguros porque, cuando se les pregunta por los factores que han contribuido a su fortalecimiento como bertsolaris, un $67 \%$ se decanta por ellas. Así, estas escuelas contribuyen a dar agencia a las mujeres bertsolaris en su empoderamiento. Aparte de ser espacios fiables, es importante el número de mujeres que concurren a ellas, es decir, que el colectivo de mujeres que asisten a esta formación es otro indicador de agencia (Kabeer, 1999). En este tránsito del poder interno al externo aparecen el feminismo y las mujeres bertsolaris referentes con un $32,8 \%$, que colaboran, apoyan y lo robustecen.

Una cuestión crucial para el tema que tratamos es el elevado abandono de las chicas, cuando han de dar el salto a la esfera pública. Un $63,9 \%$ habla de falta de seguridad o confianza, muchas por su perfeccionismo exagerado, y un $32,85 \%$ de la competitividad que supone el bertsolarismo actual; así como un 19,7\% que apunta al ambiente masculino predominante. Son los factores más destacados que marcan la retirada de este mundo.

Si se les pregunta qué se puede hacer para que las mujeres no abandonen el bertsolarismo después de una sólida formación, proponen distintos indicadores de agencia. Primeramente, hablan de la necesidad de insertar la noción de género en todo el conjunto del bertsolarismo, de formar a bertsolaris hombres y mujeres, a los organizadores, patrocinadores y jurados que intervienen en los recitales, incluir temáticas para improvisar que lo tengan en cuenta y, ¿por qué no?, también al público. También ven la necesidad de mejorar los métodos de enseñanza y, concretamente, de aprender a gestionar las emociones, controlar el miedo escénico, afianzar la seguridad y la confianza en una misma... Finalmente, de la idoneidad de crear recitales con un público cómplice, un público especializado. Todos estos indicadores de agencia suscitarían una mejor comprensión de lo que canta y cómo canta la mujer que improvisa, tanto en los innumerables festivales, concursos y eventos que se organizan, como por los miles y miles de personas que configuran las distintas audiencias del bertsolarismo.

En relación al último objetivo que nos propusimos, el de averiguar las vivencias habidas con sus cuerpos cuando saltaron a la arena pública, hemos de afirmar que coinciden al afirmar que hasta la actualidad no han realizado una reflexión consciente y explícita al respecto. Si tomamos en consideración las primeras aproximaciones al tema (Hernández, 2014; Alberdi, 2015), se señalan dos itinerarios corporales distintos. Por un lado, el inscrito por las Drag King, las mujeres que subieron en la década de los 90 a los escenarios, emulando a los hombres. Éstas se adaptaron a la coyuntura de que las mujeres podían subir al estrado, pero aceptando la masculinidad que exigía el discurso androcentrista $\mathrm{Y}$, por otro lado, la generación última, la actual, bautizada como Panpinak (muñecas). Mujeres que transgreden el orden masculino del escenario mostrando sus cuerpos femeninos, pero acatando las características del rol femenino inscrito en el patriarcado. Sin embargo, añaden que es el mercado -o el público- y su discurso hegemónico quien propone distintas formas de estar a unas y a otras y que ellas se han amoldado a dichas formas.

Por último, se preguntan dónde está el empoderamiento de su cuerpo y responden que, en el descubrimiento del mismo, como cuerpo que habla; en la reconciliación con su cuerpo y su modo de expresión; y, en la aceptación de cada cuerpo como agente de expresión e identidad. Las precursoras incorporaron inicialmente a su discurso los atributos masculinos, la pose, la rigidez y la dialéctica de la palabra, así como una voz 
potente (Hernández, 2014). Según han ido transcurriendo los años, la feminidad se ha ido apoderando de los escenarios, con un colorido transgresor. Han precisado iniciar un proceso para conocerse mejor, sus pensamientos, vivencias y emociones, para hacer frente a una audiencia aún desprovista de la perspectiva de género. Han sabido incorporar los atributos femeninos en un mundo hegemónicamente masculino, que ha castrado los cuerpos y que ha cantado desde el intelecto, del cuello para arriba. De esta manera aportan otras maneras de ser, de mirar, de estar como bertsolaris (Esteban, 2014).

\section{BiBLIOGRAFÍA}

AIERDI, Xabier (2007): Bertsolaritza tradizio modernoa, Bilbao, UPV/EHU.

AlBERDI, Uxue (2014): «Bertsolaritza isilduaz», Gorputza eta Generoa: teoria, didaktika eta esperientziak, Autoras (coor.), UEU, pp. 21-33.

Alberdi, Uxue (2015), «Bertsolaritza eta gorputza», Pikara Magazine, 20/02/2015.

Artetxe, Miren (2016): Sentitu, pentsatu, esan. Bertso-eskolak, gazteak eta portaerak, Conferencia inédita, Donostia, Capital Europea de la Cultura, Cursos de Verano.

Aubert, Adriana, Garcia, Carme, Racionero, Sandra (2009): «El aprendizaje dialógico», Cultura y educación, 21, 2, pp. 129-139. DOI: https://doi.org/10.1174/ 113564009788345826

Bourdieu, Pierre (2000), La dominación masculina, Barcelona, Anagrama.

Esteban, Mari Luz (2004): Antropología del cuerpo. Género, itinerarios corporales, identidad y cambio, Barcelona, Bellaterra.

EsteBAn, Mari Luz (2014): «Generoa, gorputza eta kultur identitate bizituaren analisia, Euskaltasuna berrirakurtzeko ahaleginean», Gorputza eta Generoa: teoria, didaktika eta esperientziak, UEU, pp. 21-33.

Freire, Pablo (1986): La pedagogía de los oprimidos, Méjico, Siglo XXI.

Foucault, Michel (1972), The Archaeology of Knowledge, New York, Pantheon Books.

Garzia, Joxerra, Sarasua, Jon, Egaña, Andoni (2001): El arte del bertsolarismo. Realidad y claves de la improvisación oral vasca, Donostia, Euskal Herriko Bertsozale Elkartea.

GARZIA, Joxerra (2012): Bertsolaritza, Donostia, Etxepare Institutua.

GonZÁlezAbrisketA, Olatz(2013): «Cuerpos desplazados. Género, deporte, y protagonismo cultural en la plaza vasca», AIBR: Revista de Antropología Iberoamericana, 8, 1, pp. 83-110. DOI: https://doi.org/10.11156/aibr.080104

Gramsci, Antonio (1971), Selections from the Prison Notebooks, Londres, Lawrence \& Wishart.

HABermas, Jürgen (1994): Teoría de la acción comunicativa, Madrid, Cátedra.

Haraway, Donna (1995): «Manifiesto para cyborgs. Ciencia, tecnología y feminismo socialista a finales del siglo XX», Simios, cyborgs y mujeres. La reinvención de la naturaleza, Madrid, Cátedra, pp. 251-311.

HERnÁNDEZ, Jone Miren (2006): «Emakume bertsolariak: ahozkatu gabeko identitatea», Kobie: antropología cultural, 12, pp. 61-70.

HERnÁNDEZ, Jone Miren (2014): «Emakume bertsolariak: bertsotik bertsora, hanka puntetan», Gorputza eta Generoa: teoria, didaktika eta esperientziak, UEU, pp. 37-48. 
KABEER, Naila (1999): «Resources, agency, achievements: Reflections on the measurement of women's empowerment», Development and Change, 30, 3, pp. 435-464. DOI: https://doi.org/10.1111/1467-7660.00125

LABORDE, Denis (1998) : «Une archéologie du bertsularisme», Antoine d'Abbadie 18971997. Congrès International, (Hendaye, 1997), Donostia, Eusko Ikaskuntza, Bilbao, Euskaltzaindia, pp. 601-620.

Larrañaga, Carmen (1995): «El Bertsolarismo: una tradición oral transitada por el género-sexo», Cuadernos de Sección. Historia y Geografía, 23, pp. 405-425.

LARRAÑAGA, Carmen (2000): «Teatralidad y poética alternativas: El bersolarismo y las mujeres», Breve historia feminista de la literatura española (en lengua catalana, gallega y vasca) Iris M. Zavala (ed.), Barcelona, Anthropos, 6, pp. 399-424.

LARrañaga Arriola, Jexux (2013): Jolas sakona: Txapelketaren prozesu errituala eta bertsolariaren arrazoi sortzailea XXI mendeko Agoran, Tesis doctoral, Bilbo, EHU/UPV. Accesible en: http://www.euskara.euskadi.net/appcont/tesisDoctoral/ PDFak/Jexux_Larranaga_TESIA.pdf

LASARTE, Gema (2012): Feminist agenda euskal narratiba garaikidean, Bilbao, EHU/ UPV.

LASARTE, Gema et al. (2016): Emakume bertsolariak: Ahanzturatik diskurtso propiora, Gasteiz, Emakunde.

LeKuona, Manuel (1978), «Idaz-lan guztiak. Aozko literatura», Kardaberaz bilduma, 22: pp. 43-73.

Lekuona, Juan Mari (1982): Ahozko euskal literatura, Donostia, Erein.

LEÓN, Magdalena (1999): «Poder y empoderamiento de las mujeres», Religión y sociedad, 11(18): pp. 189-197.

Lukes, Steven (1974): Power: A radikal Wiew, Londres, Macmillan. DOI: https://doi. org/10.1007/978-1-349-02248-9

Martínez Carazo, Piedad Cristina (2006), «El método de estudio de caso: estrategia metodológica de la investigación científica», Pensamiento \& Gestión, 20, pp. 165-193.

Mosedale, Sarah (2005): "Assessing women's empowerment: towards a conceptual framework», Journal of international development, 17, 2, pp. 243-257. DOI: https://doi.org/10.1002/jid.1212

Murguialday, Clara (2006): Empoderamiento de las mujeres: conceptualización y estrategias, Recuperado en marzo de 2017 en: http://www.vitoria-gasteiz.org/ wb021/http/contenidosEstaticos/adjuntos/es/16/23/51623.pdf

Murguialday, Clara (2013): Reflexiones feministas sobre el empoderamiento de las mujeres, Colección Cooperacció, Agencia Catalana de Cooperació al Desenvolupament.

OAKley, Peter (ed.) (2001): Evaluating Empowerment: Reviewing the Concept and Practice, Oxford, INTRAC.

Ortner, Sherry (2006): Anthropology and Social Theory: Culture, Power, and the Acting Subject, Durham, Duke University Press. DOI: https://doi.org/10.1215/ 9780822388456

PAYA, Xabier (2013): Antología de la Literatura Oral Vasca, Donostia, Instituto Vasco Etxepare.

SPIVACK, Gayatri Chakravorty (1993): «Can the Subaltern Speak? Reflections on the History of an Idea», Colonial Discourse and Post-colonial Theory: A Reader, 
Patrick Williams y Laura Chrisman (ed.), Hertfordshire, Harvester Wheatsheaf, pp. 66-111.

UGALDE, Anaisabel et al. (2020): «Mujeres vascas improvisadoras: las bertsolaris del mundo tradicional (siglos XV-XIX)», Arenal, 27, 1, pp. 141-172. DOI: http:// dx.doi.org/10.30827/arenal.v27i1.5990.

Fecha de recepción: 26 de noviembre de 2019

Fecha de aceptación: 6 de febrero de 2020

$$
9
$$

